\title{
Optimisation par colonies de fourmis pour le problème du sac à dos multidimensionnel
}

\author{
Inès Alaya, Christine Solnon, Khaled Ghédira
}

\begin{abstract}
Résumé
Dans cet article, nous proposons d'utiliser la métaheuristique d'optimisation par colonies de fourmis (Ant Colony Optimization/ACO) pour résoudre le problème du sac à dos multidimensionnel. L'objectif est de sélectionner un sous-ensemble d'objets qui maximise une fonction utilité donnée tout en respectant certaines contraintes de ressources. Dans l'algorithme ACO proposé, l'idée est de construire des solutions de façon incrémentale, par ajouts successifs d'objets à une solution partielle. A chaque itération, l'objet à ajouter est choisi selon une probabilité dépendant de traces de phéromone et d'une information heuristique locale. On étudie trois façons de déposer (et d'exploiter) les traces de phéromone : sur les objets sélectionnés, sur les couples d'objets sélectionnés consécutivement ou sur tous les couples d'objets sélectionnés. On compare le comportement de ces trois variantes sur un ensemble d'instances de référence et on étudie l'influence de la phéromone sur le processus de résolution. On compare enfin l'algorithme ACO proposé avec des approches génétiques de l'état de l'art montrant qu'il obtient des résultats compétitifs.
\end{abstract}

\section{Introduction}

Le problème du sac à dos multidimensionnel (MKP) est un problème NP-difficile qui possède de nombreuses applications pratiques comme l'allocation de processeurs dans les systèmes distribués, le chargement de cargaisons, le découpage de stocks. L'objectif du MKP est de trouver un sous-ensemble d'objets qui maximise un profit total tout en satisfaisant certaines contraintes de capacité. Plus formellement, un MKP est modélisé comme suit :

$$
\begin{aligned}
\text { maximiser } & \sum_{j=1}^{n} p_{j} x_{j} \\
\text { sous les contraintes } & \sum_{j=1}^{n} r_{i j} x_{j} \leq b_{i}, \forall i \in 1 . . m \\
& x_{j} \in\{0,1\}, \forall j \in 1 . . n
\end{aligned}
$$

où $r_{i j}$ est la quantité de ressource $i$ consommée par l'objet $j, b_{i}$ est la quantité totale disponible de la ressource $i, p_{j}$ est le profit associé à l'objet $j$, et $x_{j}$ est la variable de décision associée à l'objet $j$, i.e., $x_{j}=1$ si $j$ est sélectionné et $x_{j}=0$ sinon.

Plusieurs travaux ont été développés pour résoudre le MKP. Les méthodes exactes [BZ80, MT90, PE85, MPT80], généralement basées sur une approche par séparation et évaluation sont limitées à des instances de petites tailles, ce qui justifie le recours aux méthodes heuristiques.

\subsection{Approches heuristiques pour le MKP}

De nombreux algorithmes heuristiques ont été proposés pour résoudre le MKP. Ces algorithmes explorent l'espace de recherche du problème de façon incomplète et opportuniste; ils trouvent généralement de bonnes solutions rapidement mais ne garantissent pas l'optimalité des solutions trouvées. Beaucoup de ces algorithmes sont basés sur une approche gloutonne : partant d'un sac à dos vide, des objets sont itérativement sélectionnés en respectant un critère gradient, et en assurant la faisabilité de la solution.

D'autres algorithmes approchés combinent la programmation linéaire avec une heuristique en résolvant plusieurs relaxations du MKP. Magazine et Oguz [MO84] ont présenté un algorithme qui combine une heuristique duale avec l'approche du multiplicateur lagrangien généralisé en utilisant les multiplicateurs générés comme borne supérieure. Les heuristiques duales commencent avec une solution dont les variables sont initialisées à 1 , ensuite les variables sont mises à 0 relativement à des règles heuristiques.

Récemment plusieurs métaheuristiques ont été développées pour le MKP. Ces métaheuristiques ont permis d'avoir des résultats très compétitifs avec des instances larges. Plusieurs algorithmes basés sur la recherche taboue ont été proposées. Glover et Kochenberger [GK96] ont présenté un algorithme basé sur la recherche taboue qui utilise une mémoire flexible intégrant des informations de fréquence. 
Hanafi et Fréville [HF98] ont proposé un algorithme étroitement lié au travail de Glover et Kochenberger [GK96]. Ils trouvent des résultats meilleurs que ceux trouvés par Glover et Kochenberger sur un sousensemble des mêmes jeux de tests. Vasquez et Hao [VH01] ont présenté une approche hybride qui combine la recherche taboue avec la programmation linéaire. Cet algorithme utilise une recherche taboue qui emploie la méthode d'élimination inverse pour la gestion de la liste taboue. Cette dernière méthode a été mise en œuvre pour la première fois sur le MKP par Damneyers et Voß[DS93]. L'algorithme proposé améliore les meilleurs résultats connus jusqu'à présent sur des instances jugées difficiles.

Plusieurs algorithmes génétiques ont été développés pour le MKP, nous citons l'algorithme de Chu et Beasley [CB98] qui trouve parmi les meilleurs résultats sur des instances larges de MKP. Cet algorithme incorpore, en plus des opérateurs classiques d'un algorithme génétique, un opérateur heuristique qui utilise des connaissances spécifiques au problème.

Tout récemment trois algorithmes basés sur l'optimisation par colonies de fourmis ont été proposés. Un objectif essentiel de cet article est de comparer ces trois algorithmes.

\subsection{Optimisation par colonies de fourmis}

Les fourmis sont capables de résoudre collectivement des problèmes complexes, comme trouver le plus court chemin entre deux points dans un environnement accidenté. Pour cela, elles communiquent entre elles de façon locale et indirecte, gr ̃̃ćce à une hormone volatile, appelée phéromone : au cours de leur progression, les fourmis déposent une trace de phéromone; elles choisissent ensuite leur chemin de façon aléatoire, selon une probabilité dépendant de la quantité de phéromone précédemment déposée.

Ce mécanisme, qui permet aux fourmis de résoudre collectivement des problèmes complexes, est à l'origine des algorithmes à base de fourmis artificielles. Ces algorithmes ont été initialement proposés dans [Dor92, DMC96], comme une approche multi-agents pour résoudre des problèmes d'optimisation combinatoire. L'idée est de représenter le problème à résoudre sous la forme de la recherche d'un meilleur chemin dans un graphe, puis d'utiliser des fourmis artificielles pour rechercher de bons chemins dans ce graphe. Le comportement des fourmis artificielles est inspiré des fourmis réelles : elles déposent des traces de phéromone sur les composants du graphe et elles choisissent leurs chemins relativement aux traces de phéromone précédemment déposées ; ces traces sont évaporées au cours du temps. Intuitivement, cette communication indirecte fournit une information sur la qualité des chemins empruntés afin d'attirer les fourmis, dans les itérations futures, vers les zones correspondantes de l'espace de recherche.

Ces caractéristiques du comportement des fourmis artificielles définissent la méta-heuristique d'optimisation par une colonie de fourmis ou Ant Colony Optimization (ACO) metaheuristic [DD99, MM88, DS04]. Cette métaheuristique a permis de résoudre différents problèmes d'optimisation combinatoire, comme le problème du voyageur de commerce [DG97], le problème d'affectation quadratique [GTD99] ou le problème de routage de véhicules [BHS99].

\subsection{Motivations et organisation de l'article}

On propose dans cet article d'étudier les capacités de la métaheuristique ACO pour résoudre le problème du sac-à-dos multidimensionnel. En effet, trois algorithmes ACO pour le MKP ont été proposés récemment. Dans ces trois algorithmes, les fourmis construisent des solutions selon une heuristique gloutonne : partant d'un sac à dos vide, elles sélectionnent à chaque itération un nouvel objet, cet objet étant choisi en fonction d'un critère heuristique (caractérisant la qualité des objets candidats) et de traces de phéromone (caractérisant l'expérience passée de la colonie). Ces trois algorithmes diffèrent dans leur façon de déposer et d'exploiter les traces de phéromone : Leguizamon et Michalewizc [LM99] ont proposé de déposer des traces de phéromone sur les objets sélectionnés; Fidanova [Fid02] a proposé de déposer de la phéromone sur le chemin formé par la suite d'objets sélectionnés; Alaya, Solnon et Ghédira [ASG04] ont proposé de déposer de la phéromone sur les couples d'objets sélectionnés dans une même solution.

Un objectif essentiel de cet article est de comparer ces trois stratégies différentes de dépôt et exploitation des traces de phéromone pour résoudre le MKP avec la métaheuristique ACO. Ainsi, nous décrivons dans la section suivante l'algorithme ACO proposé pour le MKP, et nous proposons trois variantes différentes de cet algorithme, correspondant aux trois stratégies phéromonales. Nous discutons ensuite brièvement de l'influence des paramètres de l'algorithme sur l'intensification/diversification de la recherche. Enfin, nous comparons expérimentalement les trois stratégies proposées, ainsi que d'autres approches évolutionnaires, sur un ensemble de problèmes issus d'un benchmark classique.

L'intérêt de ce travail dépasse le cadre de la résolution du problème du sac à dos multidimensionnel. En effet, de nombreux problèmes d'optimisation combinatoire consistent à sélectionner un sous-ensemble d'objets optimisant une fonction objectif donnée tout en respectant un certain nombre de contraintes, e.g., la recherche de cliques maximum, les problèmes de satisfaction de contraintes. Pour résoudre ces problèmes avec la métaheuristique ACO, il s'agit essentiellement de décider sur quels composants de solutions déposer de la phéromone. Ainsi, notre étude comparative sur le MKP pourrait aider à choisir une stratégie pour la résolution d'autres problèmes de ce type. 


\section{Algorithme Ant-Knapsack :}

Initialiser les traces de pheromone a $\tau_{\max }$

repeter

pour chaque fourmi $k$ dans $1 . . n b A n t s$, construire une solution $\mathcal{S}_{k}$ comme suit :

Choisir aleatoirement un premier objet $o_{1} \in 1 . . n$

$\mathcal{S}_{k} \leftarrow\left\{o_{1}\right\}$

Candidats $\leftarrow\left\{o_{i} \in 1 . . n / o_{i}\right.$ peut etre selectionne sans violer des contraintes de ressources $\}$

tant que Candidats $\neq \emptyset$ faire

Choisir un objet $o_{i} \in$ Candidats avec la probabilite

$$
p_{S_{k}}\left(o_{i}\right)=\frac{\left[\tau_{S_{k}}\left(o_{i}\right)\right]^{\alpha} \cdot\left[\eta_{S_{k}}\left(o_{i}\right)\right]^{\beta}}{\sum_{o_{j} \in \text { Candidats }}\left[\tau_{S_{k}}\left(o_{j}\right)\right]^{\alpha} \cdot\left[\eta_{S_{k}}\left(o_{j}\right)\right]^{\beta}}
$$

$\mathcal{S}_{k} \leftarrow \mathcal{S}_{k} \cup\left\{o_{i}\right\}$

enlever de Candidats chaque objet qui viole des contraintes de ressources

\section{fin tant que}

\section{fin pour}

mettre a jour les traces de pheromone en fonction de $\left\{\mathcal{S}_{1}, \ldots, \mathcal{S}_{n b A n t s}\right\}$

si une trace de pheromone est inferieure a $\tau_{\min }$ alors la mettre a $\tau_{\min }$

si une trace de pheromone est superieure a $\tau_{\max }$ alors la mettre a $\tau_{\max }$

jusqu'a nombre maximal de cycles atteint ou solution optimale trouvee

\section{FIgURE 1 - Algorithme ACO pour le MKP}

\section{Algorithme ACO pour le MKP}

Pour résoudre le MKP avec la métaheuristique ACO, un point-clé est de décider sur quels composants des solutions construites les traces de phéromone vont être déposées et comment exploiter ces traces lors de la construction de nouvelles solutions. Une solution d'un MKP est un ensemble d'objets sélectionnés $S=\left\{o_{1}, \ldots, o_{k}\right\}$ (on considèrera qu'un objet $o_{i}$ est sélectionné si la variable de décision correspondante $x_{o_{i}}$ a été mise à 1). Etant donnée une telle solution $S$, trois différentes manières de déposer la phéromone peuvent être considérées :

- une première possibilité est de déposer les traces de phéromone sur chaque objet sélectionné dans $S$. Dans ce cas, lors de la construction des solutions suivantes, la probabilité de sélectionner chaque objet de $S$ sera augmentée,

- une deuxième possibilité est de déposer les traces de phéromone sur chaque couple $\left(o_{i}, o_{i+1}\right)$ de deux objets successivement ajoutés dans $S$. Dans ce cas, l'idée est d'augmenter la probabilité de choisir l'objet $o_{i+1}$ si le dernier objet sélectionné est $o_{i}$,

- une troisième possibilité est de déposer les traces de phéromone sur toutes les paires $\left(o_{i}, o_{j}\right)$ de deux objets appartenant à $S$. Dans ce cas, lors de la construction d'une nouvelle solution $S^{\prime}$, la probabilité de choisir l'objet $o_{i}$ sera augmentée si $o_{j}$ a déjà été sélectionné dans $S^{\prime}$. Plus précisément, plus $S^{\prime}$ contiendra de sommets appartenant déjà à $S$, et plus les autres sommets de $S$ auront de chances d'être sélectionnés.

Pour comparer ces trois stratégies phéromonales différentes, nous introduisons maintenant l'algorithme Ant-Knapsack $(\mathrm{AK})$ et nous décrivons trois variantes différentes de cet algorithme : Vertex-AK dans lequel la phéromone est déposée sur les sommets, Path-AK dans lequel la phéromone est déposée sur les arcs du chemin visité, et Edge-AK dans lequel la phéromone est déposée sur les arêtes reliant tous les sommets appartenant à une solution.

\subsection{L'algorithme Ant-Knapsack}

L'algorithme Ant-Knapsack est décrit dans la figure 1. A chaque cycle de cet algorithme, chaque fourmi construit une solution. Lorsque toutes les fourmis ont construit une solution, les traces de phéromone sont mises à jour. L'algorithme s'arrête lorsqu'une fourmi a trouvé une solution optimale (si la valeur optimale est connue), ou lorsqu'un nombre maximal de cycles a été atteint. Notons que cet algorithme est inspiré du $\mathcal{M A X}-\mathcal{M} \mathcal{I N}$ Ant System [SH00] dans lequel les traces de phéromone sont limitées à l'intervalle $\left[\tau_{\min }, \tau_{\max }\right]$ et sont initialisées à $\tau_{\max }$.

Pour construire une solution, les fourmis choisissent aléatoirement un objet initial, puis ajoutent itérativement des objets qui sont choisis à partir d'un ensemble Candidats qui contient tous les objets qui peuvent être sélectionnés sans violer de contraintes de ressources. A chaque étape, l'objet $o_{i}$ à ajouter à la solution en cours de construction $S_{k}$ est choisi parmi l'ensemble de sommets Candidats relativement à une probabilité $p_{S_{k}}\left(o_{i}\right)$. Cette probabilité est définie proportionnellement à un facteur phéromonal $\tau_{S_{k}}\left(o_{i}\right)$ et un facteur heuristique $\eta_{S_{k}}\left(o_{i}\right)$, ces deux facteurs étant pondérés par deux paramètres $\alpha$ et $\beta$ qui déterminent leur importance relative. Le facteur phéromonal dépend de la stratégie phéromonale choisie et est décrit plus tard. Le facteur heuristique $\eta_{S_{k}}\left(o_{i}\right)$ est défini de façon similaire à [LM99] et [ASG04] : soit $d_{S_{k}}(i)=b_{i}-\sum_{g \in S_{k}} r_{i g}$ 
la quantité restante de la ressource $i$ lorsque la fourmi a construit la solution $S_{k}$; nous définissons le ratio :

$$
h_{S_{k}}(j)=\sum_{i=1}^{m} \frac{r_{i j}}{d_{S_{k}}(i)}
$$

qui présente la dureté de l'objet $j$ par rapport à toutes les contraintes $i \in 1$..m et relativement à la solution construite $S_{k}$, de sorte que plus ce ratio est faible plus l'objet est intéressant. Nous intégrons alors le profit $p_{j}$ de l'objet $j$ pour obtenir un ratio profit/ressource, et nous définissons le facteur heuristique par :

$$
\eta_{S_{k}}(j)=\frac{p_{j}}{h_{S_{k}}(j)}
$$

\subsection{Définition des composants phéromonaux}

Les fourmis de l'algorithme Ant-Knapsack évoluent et déposent de la phéromone sur le graphe complet $G=(V, E)$ tel que $V$ est l'ensemble des objets. Cet algorithme s'instancie en trois versions différentes en fonction des composants du graphe sur lesquels les fourmis déposent des traces de phéromone :

- dans Vertex-AK, les fourmis déposent la phéromone sur les sommets $V$ du graphe. La quantité de phéromone sur un objet $o_{i} \in V$ est notée $\tau\left(o_{i}\right)$. Cette quantité représente la "désirabilité" de choisir l'objet $o_{i}$ lors de la construction d'une solution,

- dans Path-AK, les fourmis déposent de la phéromone sur les couples de sommets sélectionnés consécutivement, i.e. sur les arcs du graphe. La quantité de phéromone sur un arc $\left(o_{i}, o_{j}\right) \in E$ est notée $\tau\left(o_{i}, o_{j}\right)$. Cette quantité représente la "désirabilité" de choisir l'objet $o_{i}$ juste après avoir choisi l'objet $o_{j}$ lors de la construction d'une solution. Il est à noter que dans ce cas le graphe est orienté,

- dans Edge-AK, les fourmis déposent la phéromone sur les paires de sommets sélectionnés dans une même solution, i.e. sur les arêtes $E$ du graphe. La quantité de phéromone sur une arête $\left(o_{i}, o_{j}\right) \in E$ est notée $\tau\left(o_{i}, o_{j}\right)$. Cette quantité représente la désirabilité de choisir un objet $o_{i}$ lors de la construction d'une solution qui contient déjà l'objet $o_{j}$. Il est à noter que, dans ce cas le graphe est non orienté, et donc $\tau\left(o_{i}, o_{j}\right)=\tau\left(o_{j}, o_{i}\right)$.

\subsection{Définition du facteur phéromonal}

Le facteur phéromonal $\tau_{S_{k}}\left(o_{i}\right)$ traduit l'expérience passée de la colonie et est utilisé dans la probabilité de transition des fourmis pour les guider vers des zones de l'espace de recherche prometteuses. Ce facteur phéromonal depend de la quantité de phéromone déposée sur les composants phéromonaux du graphe :

- dans Vertex-AK, il dépend de la quantité déposée sur l'objet candidat, i.e.

$$
\tau_{S_{k}}\left(o_{i}\right)=\tau\left(o_{i}\right)
$$

- dans Path-AK, il dépend de la quantité présente sur l'arc connectant le dernier objet ajouté à la solution partielle $S_{k}$ et l'objet candidat $o_{i}$, i.e. si le dernier objet ajouté dans $S_{k}$ est $o_{j}$,

$$
\tau_{S_{k}}\left(o_{i}\right)=\tau\left(o_{j}, o_{i}\right)
$$

- dans Edge-AK, il dépend de la quantité déposée sur les arêtes connectant l'objet candidat $o_{i}$ avec les différents objets présents dans la solution partielle $S_{k}$, i.e.

$$
\tau_{S_{k}}\left(o_{i}\right)=\sum_{o_{j} \in S_{k}} \tau\left(o_{i}, o_{j}\right)
$$

Notons que ce facteur phéromonal peut être calculé de façon incrémentale : lorsque le premier objet $o_{1}$ a été sélectionné, le facteur phéromonal $\tau_{S_{k}}\left(o_{j}\right)$ est initialisé à $\tau\left(o_{1}, o_{j}\right)$ pour chaque objet candidat $o_{j}$; ensuite, à chaque fois qu'un nouvel objet $o_{i}$ est ajouté à la solution courante $S_{k}$, le facteur phéromonal $\tau_{S_{k}}\left(o_{j}\right)$ de chaque objet candidat $o_{j}$ est incrémenté de $\tau\left(o_{i}, o_{j}\right)$.

\subsection{Mise à jour de la phéromone}

Après que toutes les fourmis aient fini la construction de leurs solutions, les traces de phéromone sont mises à jour. Cette mise à jour s'effectue en deux étapes. Dans une première étape, toutes les traces de phéromone sont diminuées, pour simuler l'évaporation, en multipliant chaque composant phéromonal par un ratio de persistance $(1-\rho)$ tel que $0 \leq \rho \leq 1$. Notons que dans Vertex-AK, les composants phéromonaux sont associés aux objets de sorte que l'étape d'évaporation s'effectue en $\mathcal{O}(n)$ tandis que dans Path-AK et Edge-AK, les composants phéromonaux sont associés aux couples d'objets de sorte que l'étape d'évaporation s'effectue en $\mathcal{O}\left(n^{2}\right)$.

Dans un deuxième temps, la meilleure fourmi du cycle dépose de la phéromone. Plus précisément, soit $\mathcal{S}_{k} \in\left\{\mathcal{S}_{1}, \ldots, \mathcal{S}_{n b A n t s}\right\}$ la meilleure solution (celle ayant un profit maximal, les ex-aequo étant départagés aléatoirement) construite durant le cycle courant et $\mathcal{S}_{\text {best }}$ la meilleure solution construite depuis le début de 

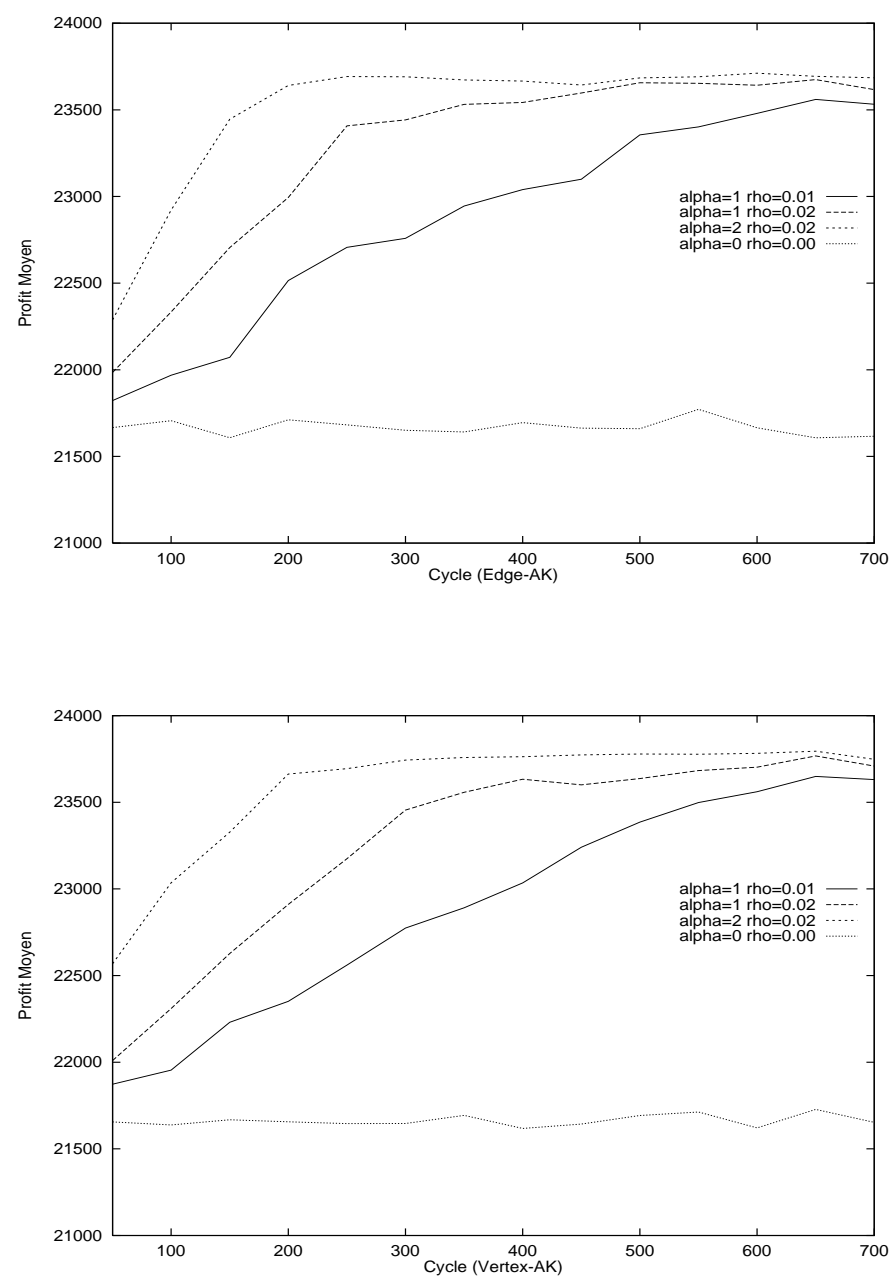

FIGURE 2 - Influence de $\alpha$ et $\rho$ sur la qualité des solutions trouvées par Edge-AK et Vertex-AK pour une instance du MKP ayant 100 objets et 5 contraintes de ressource : chaque courbe trace l'évolution du profit moyen quand le nombre de cycles augmente pour une affectation donnée de $\alpha$ et $\rho$ (moyenne sur 10 exécutions). Les autres paramètres ont été affectés à $\beta=5, n b A n t s=30, \tau_{\min }=0.01$, et $\tau_{\max }=6$

l'exécution (y compris le cycle courant). La quantité de phéromone déposée par la fourmi $k$ est inversement proportionnelle à la différence de profit entre $\mathcal{S}_{k}$ et $\mathcal{S}_{\text {best }}$, i.e. elle est égale à $1 /\left(1+\operatorname{profit}\left(S_{\text {best }}\right)-\operatorname{profit}\left(S_{k}\right)\right)$. Cette quantité de phéromone est déposée sur les composants phéromonaux de $\mathcal{S}_{k}$, i.e.

- dans Vertex-AK, elle est déposée sur chaque sommet de $\mathcal{S}_{k}$,

- dans Path-AK, elle est déposée sur les arcs du chemin visité par la fourmi $k$ lors de la construction de $\mathcal{S}_{k}$, i.e. sur tout couple de sommets $\left(o_{i}, o_{j}\right)$ tel que $o_{j}$ a été ajouté dans $S_{k}$ juste après $o_{i}$.

- dans Edge-AK, elle est déposée sur chaque arête reliant deux sommets différents de $\mathcal{S}_{k}$, i.e. sur la clique définie par $\mathcal{S}_{k}$.

Comme pour la première étape, la complexité en temps de cette deuxième étape dépend de la version considérée : pour Vertex-AK et Path-AK elle s'effectue en $\mathcal{O}\left(\left|S_{k}\right|\right)$ tandis que pour Edge-AK elle s'effectue en $\mathcal{O}\left(\left|S_{k}\right|^{2}\right)$.

\section{Influence des paramètres $\alpha$ et $\rho$ sur la résolution}

Quand on résoud un problème d'optimisation combinatoire avec une approche heuristique, il s'agit de trouver un bon compromis entre deux objectifs relativement duaux : d'une part, il s'agit d'intensifier la recherche autour des zones de l'espace de recherche les plus prometteuses, qui sont généralement proches des meilleures solutions trouvées; d'autre part, il s'agit de diversifier la recherche et favoriser l'exploration afin de découvrir de nouvelles et si possible meilleures zones de l'espace de recherche. Le comportement des fourmis par rapport à cette dualité entre intensification et diversification peut être influencé en modifiant les valeurs 
des paramètres. En particulier, la diversification peut être augmentée soit en diminuant la valeur du poids du facteur phéromonal $\alpha$ (de sorte que les fourmis deviennent moins sensibles aux traces phéromonales), soit en diminuant le taux d'évaporation $\rho$ (de sorte que la phéromone s'évapore plus doucement et les écarts d'une trace à l'autre évoluent plus doucement). Lorsque l'on augmente ainsi la capacité exploratoire des fourmis, on trouve généralement de meilleures solutions, mais en contrepartie ces solutions sont plus longues à trouver.

Nous avons pu constater que cette influence des paramètres $\alpha$ et $\rho$ sur le comportement des fourmis est identique pour les trois versions proposées de Ant-Knapsack. Nous l'illustrons dans la figure 2 pour EdgeAK et Vertex-AK. Cette figure montre que quand on augmente l'intensification, en choisissant des valeurs telles que $\alpha=2$ et $\rho=0.02$, Edge-AK trouve rapidement de bonnes solutions mais stagne plus vite sur des solutions légèrement moins bonnes. A l'inverse, en choisissant des valeurs pour $\alpha$ et $\rho$ qui favorisent l'exploration, telles que $\alpha=1$ et $\rho=0.01$, les fourmis trouvent de meilleures solutions en fin d'exécution, mais elles ont besoin de plus de cycles pour converger sur ces solutions.

Notons finalement que lorsque la phéromone n'est pas utilisée du tout, i.e. quand $\alpha=0$ et $\rho=0$, de sorte que l'algorithme se comporte comme un algorithme glouton classique, les solutions trouvées sont très nettement moins bonnes que lorsque la phéromone est utilisée.

\section{Influence des traces de phéromone sur la similarité des so- lutions calculées}

Pour expliciter l'influence de la phéromone sur la capacité des fourmis à explorer l'espace de recherche, nous proposons dans cette section de mesurer la similarité des solutions construites durant l'exécution. Plusieurs mesures de diversité ou de similarité ont été introduites pour les approches évolutionnaires, le maintien de la diversité de la population étant un point clé pour éviter une convergence prématurée et une stagnation de ces algorithmes.

Pour mesurer l'effort de diversification de Ant-Knapsack pendant l'exécution, nous proposons de calculer le taux de similarité. Ce taux correspond à la mesure de diversité de la population introduite pour les approches génétiques [MD01]. Plus précisément, le taux de similarité d'un ensemble de solutions $S$ est défini par le nombre moyen d'objets qui sont partagés par n'importe quelle paire de solutions dans $S$, divisé par le nombre moyen d'objets sélectionnés dans les solutions de $S$. Ainsi, ce taux est égal à un si toutes les solutions de $S$ sont identiques, tandis qu'il est égal à zéro si l'intersection de chaque paire de solutions de $S$ est vide.

Il est à noter que ce ratio peut être calculé très rapidement en maintenant un tableau freq tel que, pour chaque nœud $\mathrm{v}_{i} \in \mathrm{V}$, freq[i] est égal au nombre de solution de $\mathrm{S}$ qui ont sélectionné le nœud $\mathrm{v}_{i}$. Dans ce cas, le ratio de similarité de $\mathrm{S}$ est égal à $\frac{\sum_{v_{i} \in V}(\text { freq }[i] \cdot(\text { freq }[i]-1))}{(|S|-1) \cdot \sum_{S_{k} \in S}\left|S_{k}\right|}$ et il peut être calculé facilement de manière incrémentale en construisant les solutions.

Dans les algorithmes ACO, ce sont les traces de phéromone qui dirigent les fourmis vers les zones prometteuses de l'espace de recherche. La figure 3 montre l'influence de ces traces sur la similarité des solutions construites durant l'exécution de l'algorithme Ant-Knapsack, pour les deux variantes Path-AK et Edge-AK, et pour différentes valeurs des paramètres $\alpha$ et $\rho$ qui déterminent l'influence de la phéromone sur le comportement des fourmis. Ces courbes mettent en évidence trois phases dans les exécutions de Ant-Knapsack.

Durant les premiers cycles, on observe une phase d'exploration, ỗź les fourmis calculent des solutions assez différentes (les solutions construites ont $42 \%$ d'objets en commun en moyenne). La durée de cette phase d'exploration dépend du paramétrage considéré : elle est d'autant plus longue que l'on réduit l'influence de la phéromone en diminuant $\alpha$ et $\rho$. La durée de cette phase d'exploration dépend aussi de la stratégie phéromonale considérée : on observe qu'elle est plus longue pour Path-AK que pour Edge-AK. Notons que cette phase d'exploration initiale est accentuée par la stratégie du $\mathcal{M} \mathcal{A} \mathcal{X}-\mathcal{M I} \mathcal{N}$ Ant System [SH00], qui impose des bornes minimales et maximales $\tau_{\min }$ et $\tau_{\max }$ aux traces de phéromone, et initialise ces traces à $\tau_{\max }$ au début de l'exécution : ainsi, après $n$ cycles de Ant-knapsack, la trace de phéromone $\tau_{i}$ sur un composant phéromonal (arête ou sommet) $i$ est telle que $(1-\rho) \cdot \tau_{\max } \leq \tau_{i} \leq \tau_{\max }$, et comme $(1-\rho)$ est généralement très proche de 1 , les traces de phéromone ont des valeurs très similaires durant les premiers cycles et n'influencent pas significativement le calcul des probabilités de transition.

On observe ensuite une deuxième phase de convergence, ốź la similarité des solutions construites augmente progressivement, mettant en évidence le fait que les fourmis sont de plus en plus influencées par la phéromone et intensifient l'effort de recherche autour d'une zone plus petite de l'espace de recherche : la similarité des solutions construites augmente de $42 \%$ à près de $90 \%$. Notons que l'augmentation de la similarité est d'autant plus rapide que l'influence de la phéromone est forte.

On observe enfin une troisième phase de stagnation, oÃź la similarité des solutions construites se stabilise autour d'une valeur assez élevée (entre $80 \%$ et $90 \%$ ), montrant que les fourmis se sont focalisées sur une toute petite zone de l'espace de recherche qu'elles explorent intensément. 

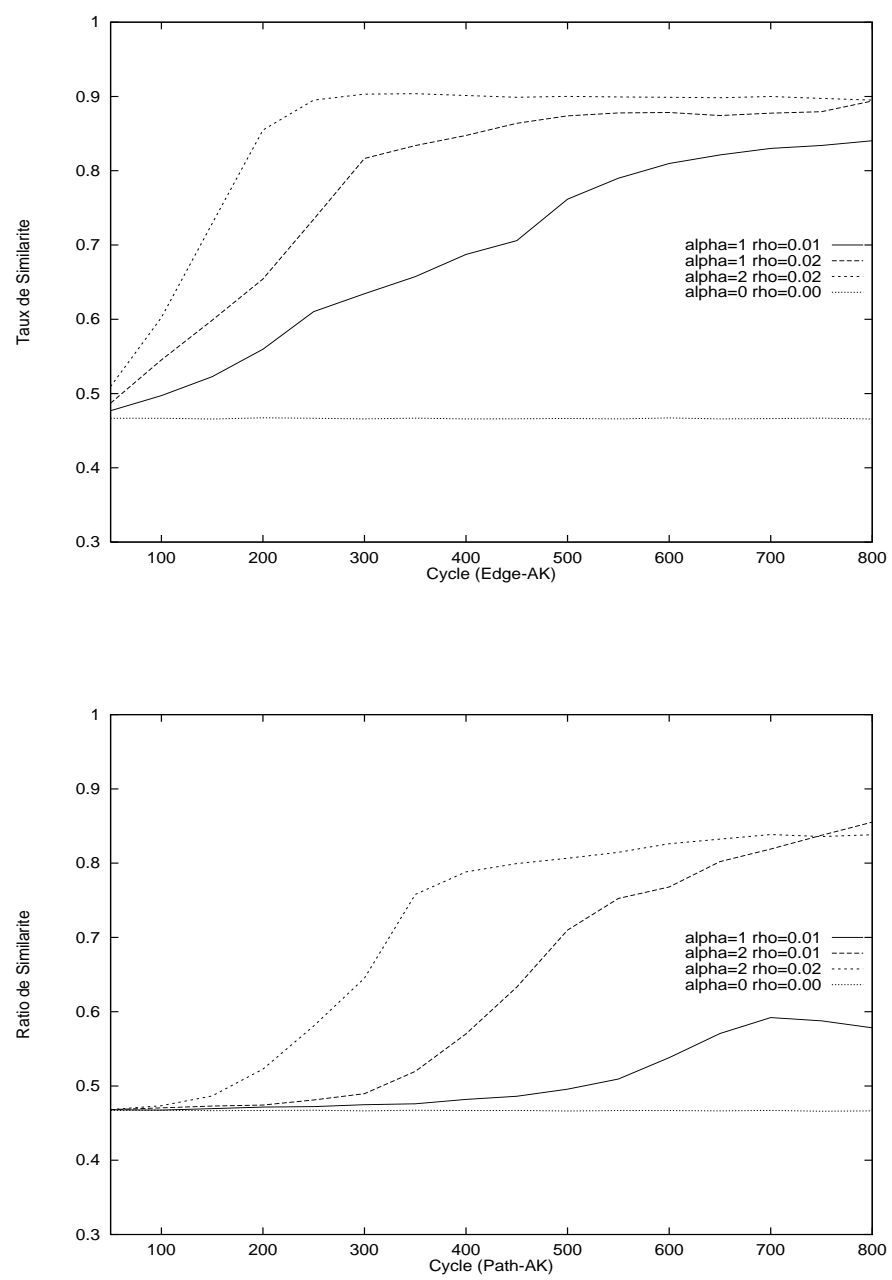

FIgURE 3 - Influence de $\alpha$ et $\rho$ sur la similarité des solutions trouvées par Edge-AK et Path-AK pour une instance du MKP ayant 100 objets et 5 contraintes de ressource : chaque courbe trace l'évolution du ratio de similarité des solutions construites quand le nombre de cycles augmente pour une affectation donnée de $\alpha$ et $\rho$ (moyenne sur 10 exécutions). Les autres paramètres ont été affectés à $\beta=5, n b$ Ants $=30, \tau_{\min }=0.01$, et $\tau_{\max }=6$

\section{Expérimentations et résultats}

Nous présentons dans cette section les résultats des trois variantes de l'algorithme Ant-Knapsack, i.e. Vertex-AK, Path-AK et Edge-AK. Nous comparons aussi ces résultats avec ceux de Leguizemon et Michalewicz [LM99] et de Fidanova [Fid02] ainsi qu'avec deux approches de l'état de l'art sur le sujet qui trouvent parmi les meilleurs résultats connus pour ces instances.

\subsection{Ensemble de tests et conditions d'expérimentation}

Nous avons considéré des instances larges de MKP OR-Library accessibles à partir du site http://www . brunel.ac.uk/dep Ces instances sont groupées en 5 classes avec $m \in\{5,10,30\}$ et $n \in\{100,250\}$. Dans la suite, ces classes sont notees m.n. Chaque classe contient 30 instances groupées en 3 sous-classes de 10 instances en fonction d'un ratio de dureté $r d: r d=0,25$ pour les 10 premières instances, $r d=0,5$ pour les 10 instances suivantes, et $r d=0,75$ pour les 10 dernières instances.

Tous les algorithmes ont été implémentés en Visual $\mathrm{C}++$ et exécutés sur un processeur PIV 2GH. Dans toutes les expérimentations, nous avons mis $\alpha$ à $1, \beta$ à $5, \rho$ à 0.01 , le nombre de fourmis à 30 et les bornes de phéromone $\tau_{\min }$ et $\tau_{\max }$ à 0.01 et 6 . Nous avons limité le nombre de cycles à 2000 cycles. 


\begin{tabular}{|cc||rr|rr|rr|}
\hline \multicolumn{1}{|c||}{$\mathrm{m} . \mathrm{n}$} & rd & \multicolumn{2}{c|}{ Vertex-AK } & \multicolumn{2}{c|}{ Path-AK } & \multicolumn{2}{c|}{ Edge-AK } \\
& & Moyenne & Ecart & Moyenne & Ecart & Moyenne & Ecart \\
\hline 5.100 & 0,25 & 24192,0 & 33,13 & 24139,3 & 149,52 & $\mathbf{2 4 1 9 7 , 2}$ & 37,52 \\
5.100 & 0,50 & 43243,3 & 24,89 & 43137,9 & 137,11 & $\mathbf{4 3 2 4 6 , 4}$ & 35,87 \\
5.100 & 0,75 & 60470,5 & 25,37 & 60435,7 & 68,86 & $\mathbf{6 0 4 7 1 , 0}$ & 28,35 \\
\hline 5.250 & 0,25 & $\mathbf{6 0 3 3 5 , 9}$ & 56,09 & 59110,6 & 911,77 & 60334,9 & 50,16 \\
5.250 & 0,50 & 109203,4 & 39,09 & 107818 & 554,38 & $\mathbf{1 0 9 2 3 1 , 6}$ & 47,97 \\
5.250 & 0,75 & 151536,6 & 36,93 & 150578,6 & 152,68 & $\mathbf{1 5 1 5 3 6 , 9}$ & 37,86 \\
\hline 10.100 & 0,25 & 22553,0 & 60,97 & 22418,2 & 193,32 & $\mathbf{2 2 5 7 2 , 2}$ & 61,81 \\
10.100 & 0,50 & 42641,0 & 50,60 & 42383,0 & 192,37 & $\mathbf{4 2 6 5 8 , 5}$ & 60,97 \\
10.100 & 0,75 & 59540,0 & 34,71 & 59464,9 & 89,42 & $\mathbf{5 9 5 5 5 , 6}$ & 44,70 \\
\hline 10.250 & 0,25 & $\mathbf{5 8 8 4 7 , 6}$ & 124,66 & 57367,3 & 593,35 & 58826,0 & 93,55 \\
10.250 & 0,50 & $\mathbf{1 0 8 6 0 0 , 7}$ & 109,50 & 106834,2 & 506,34 & 108594,1 & 78,03 \\
10.250 & 0,75 & 151272,5 & 51,03 & 150066,9 & 170,66 & $\mathbf{1 5 1 2 7 6 , 0}$ & 55,46 \\
\hline 30.100 & 0,25 & $\mathbf{2 1 6 1 8 , 1}$ & 73,33 & 21411,4 & 213,22 & 21608,4 & 75,68 \\
30.100 & 0,50 & $\mathbf{4 1 4 0 6 , 1}$ & 69,05 & 41013,0 & 228,18 & 41403,3 & 68,53 \\
30.100 & 0,75 & 59172,5 & 40,56 & 59049,9 & 111,09 & $\mathbf{5 9 1 7 3 , 2}$ & 36,67 \\
\hline \hline \multicolumn{2}{|c||}{ Moyenne } & 67642,2 & 55,33 & 67015,26 & 284,8 & $\mathbf{6 7 6 4 5 , 7}$ & 54,21 \\
\hline
\end{tabular}

TABle 1 - Comparaison de la qualité des solutions trouvées par Vertex-AK, Path-AK et Edge-AK sur les 15 classes de problèmes considérées. Chaque ligne donne les résultats pour les 10 instances de la classe $\mathrm{m} . \mathrm{n}$ ayant le ratio de dureté rd. Pour chacune de ces classes et pour chaque algorithme considéré, le tableau donne le profit trouvé (moyenne et écart type sur 10 exécutions pour chacune des 10 instances de la classe)

\subsection{Comparaison des algorithmes Vertex-AK, Path-AK et Edge-AK}

Le tableau 1 compare la qualité des résultats obtenus par Vertex-AK, Path-AK et Edge-AK sur les 15 classes de problèmes considérées. Chaque classe contient 10 instances, et chaque algorithme a été exécuté 10 fois sur chaque instance, de sorte que chaque résultat est une moyenne (ou un écart type) sur 100 exécutions.

Ce tableau montre que les deux algorithmes Vertex-AK et Edge-AK trouvent des résultats très nettement meilleurs que ceux trouvés par Path-AK, pour toutes les classes d'instances considérées. Ainsi, la stratégie consistant à déposer de la phéromone sur des objets consécutivement sélectionnés guide les fourmis de manière moins profitable que les deux autres stratégies.

En comparant Vertex-AK et Edge-AK, nous constatons que les solutions trouvées par Edge-AK sont meilleures pour 10 classes d'instances et inférieures pour les 5 autres classes. Notons que ces résultats dépendent de la taille des instances (par rapport au nombre de variables et de contraintes) : pour les instances des classes 5.100, 5.250 et 10.100, Edge-AK est clairement meilleur que Vertex-AK tandis que pour les instances des classes 10.250 et 30.100 cette tendance s'inverse.

Le tableau 2 compare les temps d'exécution et le nombre de cycles effectués par Vertex-AK, Path-AK et Edge-AK sur les 15 classes de problèmes considérées. On constate que les résultats pour les trois versions sont du même ordre de grandeur. Path-AK effectue légèrement moins de cycles et est un peu plus rapide que les deux autres versions, mais cela s'explique probablement par le fait qu'il trouve de bien moins bonnes solutions : pour Path-AK, la phéromone n'est pas capable de guider la recherche vers de bonnes zones de l'espace de recherche.

On constate que les nombres de cycles effectués par Vertex-AK et Edge-AK sont très similaires. Ce nombre dépend essentiellement du nombre de variables $\mathrm{n}$, tandis que le nombre de contraintes $\mathrm{m}$ et la dureté de ces contraintes rd ne semblent pas avoir une influence directe.

Enfin, on constate qu'un cycle de Vertex-AK est effectué légèrement plus rapidement qu'un cycle de Edge-AK. Par exemple, pour la classe 30.100 avec $r d=0,25$, les deux algorithmes ont besoin en moyenne de 810 cycles pour converger; Vertex-AK met 234 secondes pour cela tandis que Edge-AK met 316 secondes. Cette différence, qui est d'autant plus sensible que le nombre de variables n augmente, s'explique par le fait que l'évaporation est en $\mathcal{O}\left(n^{2}\right)$ pour Edge-AK alors qu'elle est en $\mathcal{O}(n)$ pour Vertex-AK.

\subsection{Comparaison avec d'autres algorithmes ACO}

L'algorithme Ant-Knapsack s'instancie en trois versions Vertex-AK, Path-AK et Edge-AK en fonction de la stratégie phéromonale choisie. L'instance Edge-AK correspond à l'algorithme décrit dans [ASG04]. Les instances Vertex-AK et Path-AK adoptent les stratégies phéromonales respectivement proposées dans [LM99] et [Fid02], mais présentent quelques différences par ailleurs. Nous comparons dans cette section les résultats obtenus par ces différents algorithmes ACO pour le MKP. 


\begin{tabular}{|c|c|c|c|c|c|c|c|c|c|c|c|c|}
\hline \multirow{3}{*}{\multicolumn{2}{|c|}{$\mathrm{m} \cdot \mathrm{n} \quad \mathrm{rd}$}} & \multicolumn{4}{|c|}{ Vertex-AK } & \multicolumn{4}{|c|}{ Path-AK } & \multicolumn{3}{|c|}{ Edge-AK } \\
\hline & & \multicolumn{2}{|c|}{ Temps } & \multicolumn{2}{|c|}{ Nb cycles } & \multicolumn{2}{|c|}{ Temps } & \multicolumn{2}{|c|}{ Nb cycles } & \multicolumn{2}{|c|}{ Temps } & Nb cycles \\
\hline & & Moy & Ec & Moy & Ec & Moy & $\mathrm{Ec}$ & Moy & Ec & Moy & Ec & Moy Ec \\
\hline 5.100 & 0,25 & 20 & 25 & 619 & 219 & 24 & 9 & 759 & 296 & 25 & 19 & 496 \\
\hline 5.100 & 0,50 & 38 & 50 & 585 & 215 & 44 & 18 & 739 & 321 & 47 & 39 & 490 \\
\hline 5.100 & 0,75 & 37 & 49 & 381 & 142 & 63 & 28 & 713 & 315 & 56 & 60 & $333 \quad 125$ \\
\hline 5.250 & 0,25 & 327 & 111 & 1062 & 357 & 182 & 97 & 720 & 384 & 343 & 89 & 1237 \\
\hline 5.250 & 0,50 & 715 & 184 & 1115 & 287 & 438 & 238 & 779 & 426 & 807 & 202 & 1321 \\
\hline 5.250 & 0,75 & 044 & 304 & 1038 & 276 & 745 & 392 & 828 & 437 & 1185 & 328 & 1197 \\
\hline .100 & 0,25 & 28 & 12 & 751 & 335 & 26 & 12 & 756 & 366 & 21 & 11 & $614 \quad 325$ \\
\hline 10.100 & 0,50 & 52 & 22 & 630 & 261 & 49 & 25 & 699 & 351 & 43 & 23 & $508 \quad 297$ \\
\hline 10.100 & 0,75 & 56 & 24 & 489 & 224 & 79 & 41 & 766 & 401 & 37 & 24 & 358 \\
\hline 10.250 & 0,25 & 373 & 172 & 1054 & 364 & 267 & 165 & 806 & 428 & 413 & 129 & 1167360 \\
\hline 10.250 & 0,50 & 813 & 276 & 1151 & 390 & 489 & 274 & 743 & 415 & 971 & 235 & 1315 \\
\hline 10.250 & 0,75 & 1232 & 396 & 1132 & 364 & 840 & 514 & 812 & 499 & 1364 & 360 & 1230 \\
\hline 30.100 & 0,25 & 234 & 250 & 808 & 325 & 93 & 81 & 774 & 309 & 316 & 248 & $810 \quad 372$ \\
\hline 30.100 & 0,50 & 284 & 291 & 749 & 302 & 113 & 56 & 769 & 386 & 400 & 213 & $797 \quad 310$ \\
\hline 30.100 & 0,75 & 262 & 292 & 515 & 188 & 162 & 78 & 853 & 428 & 437 & 267 & 587 \\
\hline \multicolumn{2}{|c|}{ Moyenne } & 368 & 164 & 805 & 283 & 241 & 135 & 768 & 384 & 431 & 150 & 831 \\
\hline
\end{tabular}

TABle 2 - Comparaison des temps d'exécution de Vertex-AK, Path-AK et Edge-AK sur les 15 classes de problèmes considérées. Chaque ligne donne les résultats pour les 10 instances de la classe $\mathrm{m} . \mathrm{n}$ ayant le ratio de dureté rd. Pour chacune de ces classes et pour chaque algorithme considéré, le tableau donne le temps (en secondes) et le nombre de cycles nécessaires pour trouver la solution (moyenne (Moy) et écart-type (Ec) sur 10 exécutions de chaque instance de la classe)

\subsubsection{Comparaison de Vertex-AK avec l'algorithme de Leguizamon et Michalewizc}

L'algorithme Vertex-AK utilise la même stratégie phéromonale que l'algorithme proposé par Leguizamon et Michalewizc dans [LM99]. Ces deux algorithmes diffèrent cependant sur les deux points suivants :

— dans l'algorithme de Leguizamon et Michalewizc, la quantité de phéromone déposée est égale au profit de la solution construite tandis que dans Vertex-AK elle est inversement proportionnelle à l'écart de profit avec la meilleure solution trouvée;

- l'algorithme de Leguizamon et Michalewiz est basé sur le schéma ACO Ant-System proposé initialement dans [DMC96] tandis que Vertex-AK est basé sur le schéma ACO MAX-MIN Ant System proposé dans [SH00].

Pour les deux algorithmes, les paramètres $\alpha$ et $\beta$, qui déterminent l'importance relative du facteur heuristique et du facteur phéromonal, ont été fixés aux mêmes valeurs (i.e. $\alpha=1$ et $\beta=5$ ). En revanche, les résultats présentés dans [LM99] ont été obtenus avec un taux d'évaporation $\rho$ fixé à 0.3 (au lieu de 0.01 pour Vertex-AK), un nombre de fourmis égal au nombre d'objets, i.e. $n$ (au lieu de 30 pour Vertex-AK) et un nombre de cycles fixé à 100 (au lieu de 2000 pour Vertex-AK).

Sur les instances des classes 5.100 et 10.100 dont le ratio de dureté est $r d=0,25$, l'algorithme de Leguizamon et Michalewiz obtient des profits de 24144 et 22457 respectivement (moyenne sur 10 exécutions pour chacune des 10 instances de la classe considérée). Si l'on compare ces résultats avec ceux de Vertex-AK (qui obtient 24192 et 22553 sur ces deux classes), on constate que Vertex-AK obtient de meilleurs résultats. Notons toutefois que sur ces instances chaque exécution de l'algorithme de Leguizamon et Michalewiz calcule $100 * 100=10000$ solutions tandis que Vertex-AK en calcule $30 * 2000=60000$.

\subsubsection{Comparaison de Path-AK avec l'algorithme de Fidanova}

L'algorithme Path-AK utilise la même stratégie phéromonale que l'algorithme proposé par Fidanova dans [Fid02]. Ces deux algorithmes diffèrent cependant sur les même points que Vertex-AK par rapport à l'algorithme de Leguizamon et Michalewizc, i.e. sur la quantité de phéromone déposée et sur le schéma ACO considéré. De plus, l'algorithme de Fidanova renforce la meilleure solution trouvée par une quantité de phéromone additionnelle. L'information heuristique dans cet algorithme est calculée de façon statique, i.e. sans prendre en compte les quantités de ressources déjà consommées par la solution en cours de construction.

Sur les instances de la classe 5.100 avec les valeurs du ratio de dureté $r d=0,25 r d=0,5$ et $r d=0,75$, l'algorithme de Fidanova obtient des profits moyens respectivement de 2393943037 et 60373. Si l'on compare ces résultats avec ceux de Path-AK (qui obtient 2413943137 et 60435), on constate que Path-AK obtient de meilleurs résultats. Notons toutefois que sur ces instances chaque exécution de l'algorithme de Fidanova calcule $200 * 100=20000$ solutions tandis que Path-AK en calcule $30 * 2000=60000$. 


\subsubsection{Comparaison de Edge-AK avec les deux algorithmes ACO}

On compare maintenant les algorithmes Edge-AK, l'algorithme de Leguizamon et Michalewizc et celui de Fidanova, qui utilise chacun une stratégie phéromonale différente. Nous trouvons que Edge-AK trouve des résultats qui sont nettement meilleurs que ceux trouvés par l'algorithme de Fidanova sur toutes les instances testées : pour la classe d'instances 5.100 l'algorithme de Fidanova trouve un profit de 42449 (moyenne des 30 instances testées) tandis que Edge-AK trouve un profit de 42638. Edge-AK trouve aussi des résultats meilleurs que ceux reportés par l'algorithme de Leguizamon et Michalewizc sur la plupart des instances testées : sur les instances des classes 5.100 et 10.100 dont le ratio de dureté est $r d=0,25$, l'algorithme de Leguizamon et Michalewiz obtient des profits de 24144 et 22457, alors que Edge-AK trouve des profits de 24197 et 60471 respectivement.

\subsection{Comparaison avec d'autres approches}

On compare maintenant Edge-AK, qui est la version de Ant-Knapsack qui obtient les meilleurs résultats en moyenne avec une approche proposée par Chu et Beasley [CB98] et une approche proposée par Gottlieb [Got00]. Ces deux approches combinent un algorithme génétique avec des techniques de réparation (pour transformer une solution violant des contraintes en une solution cohérente) et des techniques de recherche locale (pour améliorer la qualité des solutions cohérentes). Dans les deux approches la méthode de remplacement utilisée est une méthode steady state, la méthode de sélection est le tournoi binaire standard. Les deux approches ont une population de 100 individus et stoppent une exécution lorsque $10^{6}$ solutions différentes ont été générées. Les différences entre les deux approches concernent essentiellement l'opérateur de croisement et la procédure de génération de la population initiale. Il est à noter que les résultats trouvés par ces deux approches pour la plupart des instances considérées sont des valeurs optimales (pour les instances avec des valeurs optimales connues).

Le tableau 3 compare Ant-knapsack avec ces deux approches. Dans ce tableau, la qualité des solutions est mesurée par l'écart (en pourcentage) entre le profit de la meilleure solution trouvée et la valeur optimale de la relaxation du problème linéaire sur les réels, i.e. Ecart $=1-$ Best $_{\text {opt }}{ }^{L P}$ o $\tilde{A} z ́$ opt ${ }^{L P}$ est la valeur optimale du problème linéaire sur les réels et Best est la meilleure solution trouvée par l'algorithme considéré.

\begin{tabular}{|c|c|c|c|c|c|c|c|}
\hline \multicolumn{3}{|c|}{ Classe } & CB & \multicolumn{2}{|c|}{$G$} & \multicolumn{2}{|c|}{ Edge-AK } \\
\hline $\mathrm{m}$ & $\mathrm{n}$ & $r d$ & écart & écart & $\#$ sol & écart & \#sol \\
\hline \multirow[t]{3}{*}{5} & 100 & 0.25 & 0.99 & 0.99 & 74595 & 0.99 & 15736 \\
\hline & & 0.50 & 0.45 & 0.45 & 37353 & 0.47 & 16053 \\
\hline & & 0.75 & 0.32 & 0.32 & 40690 & 0.32 & 11131 \\
\hline \multicolumn{3}{|c|}{ Moyenne pour 5.100} & 0.587 & 0.587 & 50879 & 0.59 & 14306 \\
\hline \multirow[t]{3}{*}{10} & 100 & 0.25 & 1.56 & 1.60 & 190979 & 1.69 & 17874 \\
\hline & & 0.50 & 0.79 & 0.81 & 109036 & 0.79 & 17147 \\
\hline & & 0.75 & 0.48 & 0.48 & 53528 & 0.48 & 15137 \\
\hline \multicolumn{3}{|c|}{ Moyenne pour 10.100} & 0.943 & 0.965 & 117848 & 0.96 & 16919 \\
\hline
\end{tabular}

TABLE 3 - Résultats sur 6 classes de 10 instances du MKP. Pour chaque classe, on donne l'écart moyen entre la solution sur les réels et la solution trouvée par Edge-AK, Chu et Beasley (CB), et Gottlieb (G). Pour Edge-AK et Gottlieb, on reporte également le nombre moyen de solutions construites pour trouver la meilleure solution

Si l'on considère la qualité des solutions trouvées, on remarque que Edge-AK obtient les même résultats que $\mathrm{CB}$ sur quatre classes et les même résultats que G sur trois classes. Il obtient de meilleurs résultats que G sur la classe 10.100 avec $r d=0,5$. Sur les deux autres classes, il obtient des résultats légèrement moins bons. Notons toutefois que les deux algorithmes génétiques considérés utilisent des techniques de recherche locale pour améliorer la qualité des solutions construites, ce qui n'est pas le cas de Edge-AK. De plus, quand on considère le nombre de solutions construites, on remarque que Edge-AK construit au plus soixante mille solutions tandis que les deux algorithmes génétiques en construisent au plus un million. Le tableau 3 montre que G construit toujours plus de solutions que Ant-Knapsack.

\section{Conclusion}

Nous avons proposé dans cet article un algorithme ACO pour résoudre le problème du sac à dos multidimensionnel. On a proposé trois versions différentes de cet algorithme suivant les composants sur lesquels les traces de phéromone sont déposées : Vertex-AK ođ̃ź la phéromone est déposée sur les objets, Path-AK o $\tilde{A} z ́$ la phéromone est déposée sur les couples d'objets sélectionnés consécutivement, et Edge-AK oÃź la phéromone est déposée sur les paires d'objets sélectionnés dans une même solution. Un objectif principal 
était de comparer ces trois stratégies phéromonales, indépendamment des détails d'implémentation et du schéma ACO considéré.

Les expérimentations ont montré que Path-AK obtient de bien moins bons résultats que Vertex-AK et Edge-AK : pour ce problème, l'ordre dans lequel les objets sont sélectionnés n'est pas significatif, et il n'est clairement pas intéressant de ne considérer que le dernier objet sélectionné pour choisir le prochain objet à entrer dans le sac à dos. Les expérimentations ont également montré que Edge-AK obtient de meilleurs résultats que Vertex-AK sur deux tiers des classes d'instances considérées. Cependant, les différences de résultats entre ces deux versions ne sont pas aussi importantes qu'avec Path-AK.

Comme nous l'avons souligné dans l'introduction de cet article, nous pensons que cette étude expérimentale de différentes stratégies phéromonales dépasse le cadre du MKP, et peut être utile pour la résolution d'autres problèmes de recherche d'un meilleur sous-ensemble à l'aide de la métaheuristique ACO. Ainsi, pour ce genre de problème, il semble que la stratégie la plus intéressante soit de considérer l'ensemble des éléments déjà sélectionnés pour choisir le prochain élément à entrer dans le sous-ensemble, plutôt que de considérer l'intérêt de chaque élément séparément .

En comparant Edge-AK avec d'autres algorithmes ACO de l'état de l'art, qui utilisent des stratégies phéromonales différentes, on trouve qu'il donne de meilleurs résultats pour les instances considérées.

Lorsque l'on compare les résultats obtenus par Edge-AK avec d'autres approches de l'état de l'art, qui trouvent parmi les meilleurs résultats connus pour les instances testées, on constate qu'ils sont globalement compétitifs même s'ils sont légèrement moins bons sur le tiers des classes considérées. Notons toutefois qu'Edge-AK n'utilise pas de technique de recherche locale pour améliorer les solutions construites par les fourmis. Ainsi, les travaux futurs concerneront essentiellement l'intégration de telles techniques dans AntKnapsack afin de le rendre plus compétitif avec les autres algorithmes pour ce problème.

\section{Références}

[ASG04] I. Alaya, C. Solnon, and K. Ghédira. Ant algorithm for the multi-dimensional knapsack problem. In Proceedings of International Conference on Bioinspired Optimization Methods and their Applications (BIOMA 2004), pages 63-72, 2004.

[BHS99] B. Bullnheimer, R.F. Hartl, and C. Strauss. An improved ant system algorithm for the vehicle routing problem. Annals of Operations Research, 89 :319-328, 1999.

[BZ80] E. Balas and E. Zemel. An algorithm for large zero-one knapsack problems. Operations Research, $28: 1130-1145,1980$.

[CB98] P.C. Chu and J.E. Beasley. A genetic algorithm for the multidimentional knapsack problem. Journal of heuristics, 4 :63-86, 1998.

[DD99] M. Dorigo and G. Di Caro. The Ant Colony Optimization meta-heuristic. In D. Corne, M. Dorigo, and F. Glover, editors, New Ideas in Optimization, pages 11-32. McGraw Hill, UK, 1999.

[DG97] M. Dorigo and L.M. Gambardella. Ant colony system : A cooperative learning approach to the traveling salesman problem. IEEE Transactions on Evolutionary Computation, 1(1) :53-66, 1997.

[DMC96] M. Dorigo, V. Maniezzo, and A. Colorni. The Ant System : Optimization by a colony of cooperating agents. IEEE Transactions on Systems, Man, and Cybernetics - Part B, 26(1) :29-41, 1996.

[Dor92] M. Dorigo. Optimization, Learning and Natural Algorithms (in Italian). PhD thesis, Dipartimento di Elettronica, Politecnico di Milano, Italy, 1992.

[DS93] F. Dammeyer and S.Voss. Dynamic tabu list management using the reverse elimination method. Annals of Operations Research, 41 :31-46, 1993.

[DS04] M. Dorigo and T. Stüzle. Ant Colony Optimization. Springer-Verlag, 2004.

[Fid02] S. Fidanova. Evolutionary algorithm for multidimensional knapsack problem. In Proceedings of PPSNVII, 2002.

[GK96] F. Glover and G.A. Kochenberger. Critical event tabu search for multidimensional knapsack problems. In I.H.Osman and J.P.Kelly, editors, Metaheuristics : the Theory and Applications, pages 407-427. Kluwer Academic Publishers, Dordrecht, The Netherlands, 1996.

[Got00] J. Gottlieb. Permutation-based evolutionary algorithms for multidimensional knapsack problems. In Proceedings of the 2000 ACM symposium on Applied computing, pages 408-414, 2000.

[GTD99] L. Gambardella, E. Taillard, and M. Dorigo. Ant colonies for the quadratic assignment problem. Journal of the Operational Research Society, 50 :167-176, 1999.

[HF98] S. Hanafi and A. Fréville. An approximate algorithm for multidimentional zero-one knapsack problems a parametric approach. Management Science, 43 :402-410, 1998.

[LM99] G. Leguizamon and Z. Michalewicz. A new version of ant system for subset problem. In Proceedings of Congress on Evolutionary Computation, pages 1459-1464, 1999.

[MD01] R.W Morrison and K.A DeJong. Measurement of population diversity. In In 5th International Conference EA 2001, volume 2310 of LNCS, pages 31-41. Springer-Verlag, 2001. 
[MM88] Moyson and Manderick. The collective behaviour of ants : an example of self organisation in massive parallelism. In Proceedings of AAAI Spring Symposium on Parallel Models of Intelligence, 1988.

[MO84] M.J. Magazine and O. Oguz. A heuristic algorithm for the multidimensional zero-one knapsack problem. European Journal of Operational Research, 16 :319-326, 1984.

[MPT80] S. Martello, D. Pisinger, and P. Toth. New trends in exact algorithms for the 0-1 knapsack problems. Europeen Journal of Operational Research, 123 :325-336, 1980.

[MT90] S. Martello and P. Toth. Knapsack Problems : Algorithms and computer implementations, in : Series in Discrete Mathematics and Optimization. Wiley Inter Sciences, New York, 1990.

[PE85] G. Plateau and M. Elkihel. A hybrid method for the 0-1 knapsack problem. Methods of Operations Research, $49: 277-293,1985$.

[SH00] T. Stützle and H.H. Hoos. $\mathcal{M} \mathcal{A} \mathcal{X}-\mathcal{M I N}$ Ant System. Journal of Future Generation Computer Systems, 16 :889-914, 2000.

[VH01] M. Vasquez and J.K Hao. A hybrid approach for the 0-1 multidimensional knapsack problem. In Proceedings of IJCAI'01, pages 328-333, 2001. 